Den Energieverlust durch Stöße zwischen Plasmateilchen und Neutralteilchen (also im wesentlichen zwischen Metall-Ionen und Wasserstoffatomen) läßt sich so abschätzen: pro Stoß wird der Bruchteil $m_{\mathrm{H}} / m_{\mathrm{i}} \approx 10^{-1}$ der kinetischen Energie eines Wasserstoffatoms umgesetzt, mit der im Abschnitt (IXc) angegebenen Stoßhäufigkeit folgt also als Energiedissipation

$$
\left(m_{\mathrm{H}}^{2} / 2 m_{\mathrm{i}}\right) q N_{0} N_{\mathrm{i}}(\Delta V)^{3} \quad\left[\mathrm{erg} \mathrm{cm}^{-3} \mathrm{sec}^{-1}\right]
$$

und um die Leistungsdichte von $10^{-24} \mathrm{erg} \mathrm{cm}^{-3} \mathrm{sec}^{-1}$ umzusetzen, ist eine Geschwindigkeitsdifferenz $\Delta \mathrm{V}$ $\approx 10^{6} \mathrm{~cm} \mathrm{sec}^{-1}$ erforderlich. Wir hatten aber für die tatsächliche Größe von $\Delta V$ den Wert $10^{4.4} \mathrm{~cm} \mathrm{sec}^{-1}$ abgeschätzt. Danach sollte i. allg. der Beitrag der ambipolaren Diffusion zur Energiedissipation unerheblich sein. Bei den starken lokalen Schwankungen der eingehenden Größen mag er jedoch in einzelnen Bereichen wesentlich sein.

Den HHrn. O. H a x e l, C. F. v. We izsäcker und G. K. B a t c h e lor sind wir für wichtige Anregungen zu großem Dank verpflichtet. Ganz besonders möchten wir Hrn. W. He is e n berg danken für sein großes Interesse und zahlreiche Diskussionen.

\title{
Zur Quantentheorie der Elementarteilchen
}

\author{
Von W. Heisenberg \\ Aus dem Max-Planck-Institut für Physik, Göttingen \\ (Z. Naturforschg. 5 a, 251-259 [1950]; eingegangen am 23. Februar 1950)
}

\begin{abstract}
Die Fortschritte, die in den letzten Jahren in der Quantentheorie der Wellenfelder erzielt worden sind, genügen, um ein mathematisches Schema anzugeben, nach dem die zukünftige Theorie der Elementarteilchen möglicherweise konstruiert ist. Dieses Schema hat die folgenden Eigenschaften: Die Gesamtheit aller Elementarteilchen wird durch ein einziges Spinorfeld dargestellt. Dieses Spinorfeld genügt (in der sogenannten Wechselwirkungsdarstellung) einer im ganzen Raum-Zeit-Gebiet regulären Vertauschungsrelation (mit positivem Zeichen); ferner wird eine relativistische Invariante als Wechselwirkungsenergie (ein Ausdruck mindestens 4. Grades im Spinorfeld) vorgegeben. Diese beiden Funktionen, die Vertauschungsfunktion und die Wechselwirkungsenergie, charakterisieren die Theorie vollständig. Aus ihnen lassen sich die Ruhmassen aller Elementarteilchen grundsätzlich berechnen, wobei eine reinliche Scheidung zwischen Elementarteilchen und zusammengesetzten Teilchen unmöglich ist. Aus diesem Grunde kommen im allgemeinen Elementarteilchen sowohl mit ganzzahligen wie mit halbzahligen Spinwerten (dementsprechend Bose- bzw. Fermi-Statistik) vor; auch die Lichtquanten sind durch das Spinorfeld darzustellen. Bei der Berechnung der Ruhmassen oder der Wechselwirkung der Elementarteilchen treten wegen cier regulären Vertauschungsrelation keinerlei divergente Ausdrücke auf, vielmehr verhält sich die ganze Theorie mathematisch ebenso regulär wie die gewöhnliche unrelativistische Quantenmechanik. Diese Eigenschaften scheinen dem Verf. ein starkes Argument dafür, daß die spätere Theorie der Elementarteilchen, die man dann auch eine „einheitliche Feldtheorie“ nennen könnte, nach einem derartigen Schema konstruiert ist.
\end{abstract}

$\mathrm{D}$ ie großen Fortschritte, die in der Quantentheorie der Wellenfelder in den letzten Jahren erzielt worden sind, haben verschiedene Bausteine für eine künftige Theorie der Elementarteilchen zusammengetragen. Zunächst ist der mathematische Apparat

1 S. Tomona ga, Progress theor. Physics 1, 27 [1946]; Koba, Tati u. S. Tomonaga, ibid. 2, 101, 198 [1947]; S. Kanes awa u. S. Tomonaga, ibid. 3, 1, 101 [1948] u. S. To m o n a g a, Physic. Rev. 74, 224 [1948]; vgl. auch W. H e is en berg, Z. Physik 110, 251 [1938].

2 R. P. F e y n m a n, Rev. mod. Physics 20, 367 [1948]; Physic. Rev. 74, 939, 1430 [1948]; J. W h e e le r u. R. P. F e y n m an, Rev. mod. Physics 17, 157 [1945].

3 J. S c h w in g e r, Physic. Rev. 74, 1439 [1948]; 75, 651 [1949]; 75, 790 [1949]. zur durchgehend relativistischen Behandlung einer Feldtheorie durch die Arbeiten von Tom on a g a ${ }^{1}$, Feynman ${ }^{2}$, S chwinger ${ }^{3}$ und Dy s on ${ }^{4}$ weitgehend vervollkommnet worden. Dann haben B opp ${ }^{5}$, Stückelberg ${ }^{6}, \mathrm{Pais}^{7}, \mathrm{Pauli}$ und Villars ${ }^{8}$

4 F. J. D y s o n, Physic. Rev. 75, 486, 1736 [1949].

5 F. B o p p, Ann. Physik 42, 575, u. 43, 565 [1943]; Z. Naturforschg. 1, 53, 237 [1946].

${ }_{6}^{6}$ E. C. St ü c k el be r g, Nature 144, 118 [1939]; Helv. physica Acta 14, 51 [1941]; E. C. S t ü c k e l b e r g u. D. Rivier, Physic. Rev. 74, 218, 986 [1948]; D. R ivie r, Helv. physica Acta 22, 265 [1949].

I A. P a is, The Development of the Theory of the Electron, Princeton Univ. Press, 1948.

8 W. P a u li u. F. V illars, Rev. mod. Physics 21, 434 [1949]. 
und andere gezeigt, daß die Singularitäten in den üblichen Feldtheorien wesentlich gemildert oder beseitigt werden können, wenn man die Differentialgleichungen durch allgemeinere Integro-Differentialgleichungen ersetzt. Die Untersuchungen $\mathrm{S} \mathrm{chw}$ in gers ${ }^{3}$ und Dys ons ${ }^{4}$ zur Quantenelektrodynamik haben gelehrt, wie man mit den nichtlinearen Wechselwirkungstermen einer Feldtheorie umzugehen hat und wie die korrespondenzmäßig-anschauliche Darstellung dann erst nach Durchführung gewisser Renormalisierungen zum Vorschein kommt. Dabei ist auch die Verbindung zum S-Matrix-Formalismus ${ }^{9}$ hergestellt worden, der zunächst unabhängig von der Quantenelektrodynamik entwickelt worden war. Schließlich ist von B o p p und anderen ${ }^{10}$ die Brücke geschlagen worden von den verallgemeinerten Integralgleichungen einer linearen Feldtheorie zu den Bewegungsgleichungen einzelner Elementarteilchen mit beliebigem Spin. Diese verschiedenen Bausteine sind aber bisher noch nicht zu einem einheitlichen Gebäude zusammengefügt worden.

Die physikalischen Gesichtspunkte für eine Theorie der Elementarteilchen wurden in zwei früheren Arbeiten ${ }^{9 \text { u. } 11}$ des Verf. besprochen, die die Grundlage für die folgenden Überlegungen abgeben sollen. Dort wurde hervorgehoben, daß die Unterscheidung zwischen Elementarteilchen und zusammengesetzten Teilchen grundsätzlich wohl kaum aufrecht erhalten werden kann und daß sich alle Elementarteilchen aus einer primären Hamilton-Funktion sollten ableiten lassen, sofern der Hamilton-Formalismus überhaupt brauchbar ist. Dann wurde vermutet, daß ein Formalismus, der dies leistet, irgendwo in der Mitte liegen sollte zwischen einem mit einer nichtlinearen Integralgleichung arbeitenden Hamilton-Formalismus und einem Gleichungssystem, das die S-Matrix unmittelbar, ohne Umweg über eine Hamilton-Funktion, bestimmt.

Die verschiedenen Fortschritte der letzten Jahre machen es nun wahrscheinlich, daß diese Grenzen ziemlich eng beisammen liegen und daß eine nichtlineare Integralgleichung, die zur Berechnung der $S$-Matrix, eventuell unter geringer Verallgemeinerung des Hamilton-Formalismus, benutzt wird, tatsächlich

9 W. H e is e n b e r g, Z. Physik 120, 513, 673 [1943]; Z. Naturforschg. 1, 608 [1946]; Chr. M öll e r, Verh. kgl. dän. Akad. Wiss. 23, 1 [1945]; 24, 19 [1946]; K. W ild er muth, Z. Physik 127, 85, 92 u. 122 [1949].

10 F. B o p p, Z. Naturforschg. 3a, 564 [1948]; W. W ess el, Z. Naturforschg. 3 a, 559 [1948]. Weitere Literaturangaben in diesen beiden Arbeiten.

11 W. He is e n berg, Two Lectures. Cambridge Univ. Press 1949. schon eine geschlossene mathematische Theorie ermöglicht, die reichhaltig genug ist, um die Gesamtheit aller Elementarteilchen darzustellen. Dies soll im folgenden an einem Modell einer solchen Theorie gezeigt werden, das dann zugleich die in den beiden genannten Arbeiten besprochenen physikalischen $\mathrm{Ge}$ sichtspunkte näher erläutert.

\section{Die Grundgleichungen des Modells}

Der Zustand des Feldes werde beschrieben durch einen Spinor $\psi_{\alpha}(x)$, für den eine Vertauschungsrelation mit positivem Vorzeichen gelten soll. (In der Bezeichnung schließen wir uns an die Arbeiten voin Schwinger ${ }^{3}$ an.) Daß hier an die Spitze der Theorie eine Spinor, nicht ein an sich einfacherer Skalar gesetzt wird, hat zwei Gründe: Erstens kann man die Gesamtheit der Elementarteilchen nur mit einem Spinor, nicht mit einem Skalar darzustellen hoffen; denn man kann zwar aus einem Spinor Skalare und Vektoren bilden, nicht aber aus einem Skalar einen Spinor. Zweitens fällt bei den Spinoren, zwischen denen eine Vertauschungsrelation (V.R.) mit positivem Vorzeichen besteht, eine Schwierigkeit weg, die mit den „Quanten negativer Energie“ zusammenhängt und später besprochen werden soll.

Ferner benützen wir im folgenden stets die sogenannte Wechselwirkungsdarstellung (Bezeichnung nach $\mathrm{S}$ chwinge ${ }^{3}$ ), in der die V.R. der Wellenfunktion zu beliebigen Raum-Zeitpunkten festgelegt werden; diese Darstellung hat den doppelten Vorteil, die relativistische Invarianz in jedem Schritt der Rechnung zu zeigen und den Anschluß an den S-MatrixFormalismus zu erleichtern.

Wir setzen also

$$
\psi_{\alpha}(x) \psi_{\beta}(x)^{\prime}+\psi_{\beta}\left(x^{\prime}\right) \psi_{\alpha}(x)=-i S_{\alpha_{\beta}^{3}}^{R}\left(x-x^{\prime}\right) .
$$

Die Funktion $S_{a, 3}^{R}$ (die nicht mit der S-Matrix verwechselt werden darf!) soll eine im Sinne der Arbeiten von Feynman ${ }^{2}, \mathrm{~S}$ t ü ckel berg und Rivier ${ }^{6}$, $\mathrm{P}$ a uli und Villars ${ }^{8}$ regularisierte Vertauschungsfunktion sein, die keine Singularitäten aufweist. Dabei stellen wir uns nicht auf den „formalistischen“ Standpunkt von Pauli und Villars, sondern auf den (nach dem Sprachgebrauch von Pauli und Villars) „realistischen“" von Bopp ${ }^{5}$ und Fey $\mathrm{mman}^{2}$; d. h. es sollen an der Funktion $S_{u, 3}^{R}$ später keinerlei Grenzübergänge und dgl. ausgeführt werden. Diese Funktion charakterisiert vielmehr die ganze Theorie und bestimmt, sofern von der Wechselwirkung abgesehen wird, welche Elementarteilchen es geben soll; die 
Funktion $S_{\alpha \beta}^{R}$ enthält als fundamentale Konstante eine Länge von der Größenordnung $10^{-13} \mathrm{~cm}$, sie bezeichnet also die Stelle, an der die sog. „kleinste Länge" 12 in die Theorie eingeführt wird.

Der Zusammenhang zwischen Gl. (1) und der linearen Integralgleichung, der $\psi_{\boldsymbol{\alpha}}(x)$ bei Vernachlässigung der Wechselwirkung genügt, ist bei Bo $\mathrm{p} \mathrm{p}^{5}$, $\mathrm{St}$ ü $\mathrm{ckel}$ e e r $\mathrm{g}^{6}$ und $\mathrm{F}$ e y $\mathrm{n} \mathrm{m}$ a $\mathrm{n}^{2}$ und neuerdings insbesondere von $\mathrm{Pa}$ is und $\mathrm{Uhlenbek^{13 }}$ ausführlich untersucht worden. Er sei hier für den Spinorfall kurz skizziert.

Wir setzen mit $\mathrm{S} c \mathrm{~h}$ wing e r

$$
S_{\alpha \beta}^{\varkappa}\left(x-x^{\prime}\right)=(2 \pi)^{-4} \int d k \frac{i k_{\tau} \gamma_{\tau}^{\alpha^{3}}-\varkappa}{k_{\lambda}{ }^{2}+\varkappa^{2}} e^{i k_{\sigma}\left(x-x^{\prime}\right)_{\sigma}} .
$$

Ferner sei, unter Einführung eines Massenspektrums $\varrho(\varkappa):$

$$
1 / \zeta(k)=\int \varrho(\varkappa) d \% \frac{i k_{\tau} \gamma_{\tau}-\varkappa}{k_{\lambda}^{2}+\varkappa^{2}} \text {, und }
$$

$$
\begin{aligned}
S_{\alpha \beta}^{R}\left(x-x^{\prime}\right)=\int \varrho(x) d \varkappa S_{\alpha \beta}^{\varkappa}\left(x-x^{\prime}\right) & \\
& =(2 \pi)^{-4} \int d k \frac{e^{i k_{\sigma}\left(x-x^{\prime}\right)_{\sigma}}}{\zeta(k)} .
\end{aligned}
$$

Die zugehörige Wellenfunktion $\psi_{\alpha}$ genügt der Wellengleichung

$$
\zeta(k) \psi(k)=\bar{\psi}(k) \bar{\zeta}(k)=0
$$

oder im Ortsraum

$$
\begin{aligned}
& \int \varepsilon\left(x-x^{\prime}\right) \psi\left(x^{\prime}\right) d x^{\prime}=0, \\
& \text { wobei } \quad \varepsilon\left(x-x^{\prime}\right)=\int \zeta(k) d k e^{i k_{\mu}\left(x-x^{\prime}\right) \mu} .
\end{aligned}
$$

Die Vertauschungsfunktion $S^{R}$ ist nur dann regulär, wenn

$$
\int \varrho(x) \varkappa^{n} d x=0 \text { für } n=0,1,2,3 .
$$

Speziell kann das Massenspektrum $\varrho(\varkappa)$ diskret sein. Dann ist

$$
\varrho(\%)=\sum_{l} c_{l} \delta\left(\%-\varkappa_{l}\right)
$$

[z. B. kann einfach $\zeta(k)=\prod_{l}\left(i k_{\tau} \gamma_{\tau}+x_{l}\right)$ gelten]. Aus den Bedingungen (4) folgt in diesem Fall, daß die $c_{l}$ zum Teil positiv, zum Teil negativ sein müssen. Diese Tatsache führte bei Bopp und Stückelberg zu der bekannten Schwierigkeit, daß einige Quanten (nämlich die zu den negativen $c_{l}$ gehörigen) negative Energien

12 W. Heis e nberg, Ann. Physik 32, 20 [1938].

13 A. $\mathrm{P}$ a is u. G. E. U h le n b e c k, On Field Theories with Non-Localized Action.

Für die Möglichkeit, diese Arbeit vor der Drucklegung kennenzulernen, bin ich den Herren Pais und Uhlenbeck zu großem Dank verpflichtet. erhielten. Hier im Spinorfall kann diese Schwierigkeit nicht auftreten, da man ja bei Gültigkeit der FermiStatistik die negativen Energien stets durch Anwendung der Löchervorstellung umdeuten kann. Dafür entsteht hier aber die andere Schwierigkeit, daß man $\bar{\psi}$ nicht einfach als die (im Sinne der Diracschen Theorie) zu $\psi$ hermitisch zugeordnete Größe definieren kann. Setzt man nämlich

$$
\psi=\sum \alpha_{l} \psi_{l} \quad \text { und } \quad \bar{\psi}=\sum \alpha_{l} \bar{\psi}_{l},
$$

wobei $\psi_{l}$ jeweils die Teilchensorte mit der Masse $\varkappa_{l}$ darstellt und $\bar{\psi}_{l}$ hermitisch zugeordnet zu $\psi_{l}$ ist, so folgt $\alpha_{l}^{2}=c_{l}, \mathrm{~d}$. h. die $\alpha_{l}$ müssen imaginär sein, wenn $c_{l}$ negativ ist. Dann ist aber $\bar{\psi}=\Sigma \alpha_{l} \bar{\psi} l$ nicht mehr hermitisch zugeordnet zu $\psi$. Wir setzen also für diese negativen $c_{l}$-Werte: $\alpha_{l}=i \beta_{l}$, wobei $j^{2}=-1$ ist, und schreiben

$\psi=\sum_{c_{l}>0} \alpha_{l} \psi_{l} \dot{+} i \sum_{c_{l}<0} \beta_{l} \psi_{l} ; \bar{\psi}=\sum_{c_{l}>0} \bar{a}_{l} \bar{\psi}_{l}+i \sum_{c_{l}<0} \bar{\beta}_{l} \bar{\psi}_{l}$.

Dann kann man sagen, daß die $\bar{\psi}$ „bis auf den Vorzeichenwechsel von $j^{\text {“ zu }} \psi$ hermitisch zugeordnet seien.

Aus dem Ansatz (1) wird eine Theorie von Elementarteilchen erst, wenn man diesen Ansatz durch Angabe einer (relativistisch invarianten) Wechselwirkungsenergie $H(x)$ vervollständigt. Z. B. könnte man an einen Ansatz von der Form

$$
H(x)=A\left(\bar{\psi}(x) \gamma_{\mu} \psi(x)\right)\left(\bar{\psi}(x) \gamma_{\mu} \psi(x)\right)
$$

denken. Allerdings wäre dieser Ausdruck noch nicht hermitisch und daher für die Wechselwirkung in dieser Form noch nicht zu brauchen*. Wir wollen aber an der Forderung festhalten, daß $H(x)$ jedenfalls als Funktion von $\bar{\psi}$ und $\psi$ dargestellt werden soll. Dann muß man nach speziellen Ausdrücken suchen, die gegenüber einer Vorzeichenänderung von $i$ invariant sind. Wenn die Wechselwirkung sich in der üblichen Weise durch die $\bar{\psi}$ und $\psi$ ausdrücken läßt und außerdem gegenüber der Vorzeichenänderung von $j$ invariant ist, so ist sie auch hermitisch. Wir verschieben die Frage, ob solche Ausdrücke gebildet werden können, auf später und untersuchen zunächst die Wirkung von $H(x)$.

* Auf die Schwierigkeiten, die hier mit der Hermitizitätsforderung verbunden sind und die im ersten Entwurf der Arbeit noch nicht beachtet waren, hat mich Herr W.Pauli freundlicherweise aufmerksam gemacht, dem ich hierfür zu großem Dank verpflichtet bin. Zur Klärung dieser Schwierigkeiten ist der Absatz II d bei der Korrektur zugefügt worden. 
Die Einführung der Wechselwirkung $H(x)$ bringt die folgenden Veränderungen hervor:

Erstens fügt sie zu den Ruhmassen $\varkappa_{i}$ der Elementarteilchen eine im allgemeinen nicht verschwindende und von $\varkappa_{i}$ abhängige Selbstenergie; sie verändert also das Massenspektrum. Zweitens kann sie dazu führen, daß zwei oder mehr Teilchen sich zu zusammengesetzten Teilchen verbinden, daß also neue Teilchen entstehen. Dabei wird man die Unterscheidung zwischen zusammengesetzten und einfachen Teilchen nur treffen können, solange die Konstante $A$ sehr klein ist $\left(A \ll l^{2}\right.$, wenn $l$ die „kleinste Länge“ bedeutet). Für große Werte von $A\left(A \gtrsim l^{2}\right)$ erhält die Bindungsenergie die gleiche Größenordnung wie die ursprüngliche Ruhmasse der einzelnen Teilchen. Es entstehen also neue „Elementarteilchen“, die im Gegensatz zu den Ausgangsteilchen auch ganzzahlige Spinwerte haben können. Insbesondere können, etwa durch eine genaue Kompensation der Ruhmassen mit der Wechselwirkungs- und Selbstenergie, aus einer geraden Anzahl von Spinorteilchen auch z. B. Photonen gebildet werden. Drittens erzeugt die Funktion $H(x)$ eben eine Wechselwirkung der Teilchen (der „einfachen“ sowohl wie der „zusammengesetzten“), die infolge von $H(x)$ aneinander gestreut, emittiert oder absorbiert werden können.

Die Aussagen über Streuung, Emission und $\mathrm{Ab}$ sorption werden alle zusammengefaßt in der $S$ Matrix, die nach Dy s o ${ }^{4}$ aus $H(x)$ nach der Formel berechnet werden kann:

$$
\begin{aligned}
S=\sum_{n=0}^{\infty} \frac{(-i)^{n}}{n !} \int_{-\infty}^{+\infty} d x_{1} \ldots \int_{-\infty}^{+\infty} d x_{n} \\
\cdot P\left(H\left(x_{1}\right), H\left(x_{2}\right) \ldots H\left(x_{n}\right)\right) .
\end{aligned}
$$

Dabei bedeutet $P$ das Produkt der $H(x)$-Funktionen in einer solchen Reihenfolge, daß frühere $x$-Werte stets rechts von späteren stehen. Die S-Matrix kann also berechnet werden, wenn die Wechselwirkung $H(x)$ und die Vertauschungsrelationen gegeben sind.

Läßt man das Zeichen $P$ weg, nimmt also das Produkt mit einer ein- für allemal festen Reihenfolge, so entsteht übrigens aus (9) einfach die aus früheren Darstellungen der S-Matrix bekannte Formel ${ }^{9}$

$$
S=e^{i \eta}
$$

wobei $\eta=-\int_{-\infty}^{+\infty} H(x) d x$ ist. Es muß aber betont wer-

den, daß die Formel (9) reichhaltiger ist als (10). Zum Beispiel verschwindet die Selbstenergie bei (10) in der Regel, während sie bei (9) im allgemeinen nicht verschwindet. Andererseits ist die Berechnung von $S$ bei Annahme von (10) sehr viel einfacher als bei Gl. (9), da man $S$ in Gl. (10) am besten dadurch bestimmt, daß man $\eta$ auf Diagonalform bringt. Einige Beispiele sind nach diesem Verfahren in einer früheren Arbeit ${ }^{9}$ durchgerechnet worden.

Wir fassen also zusammen: Durch die beiden Funktionen $S_{\alpha \beta}^{R}\left(x-x^{\prime}\right)$ [oder $\varrho(x)$ ] und $H(x)$ wird eine Theorie der Elementarteilchen festgelegt, in der es eine Reihe von verschiedenen Teilchen mit ganz- oder halbzahligen Spinwerten gibt. Ob diese Teilchen als „elementar“ oder als „zusammengesetzt“ bezeichnet werden sollen, ist eine von den quantitativen Verhältnissen abhängige Zweckmäßigkeitsfrage. Wenn gezeigt werden kann, daß eine derartige Theorie überall konvergente Resultate ergibt, d. h. alle Divergenzschwierigkeiten vermeidet, so scheint es plausibel, anzunehmen, daß die eigentliche Theorie der Elementarteilchen eine derartige Form hat. Alle Elementarteilchen würden dann als Zustände ein und derselben Grundmaterie erscheinen. Insofern verwirklicht eine solche Theorie genau das in der früheren Arbeit erörterte Programm.

II. Mathematische Untersuchungen über die Eigenschaften des Modells

a) Energie und Impuls im Grenzfall $A=0$

Die Frage nach dem Energie-Impulstensor in einer Integralgleichungstheorie, die durch eine Funktion $\zeta(k)$ im Sinne von Gl. (3) charakterisiert wird, ist schon früher ausführlich untersucht worden. Dabei hatte sich herausgestellt, daß man bei Übergang vom Orts- zım Impulsraum ohne Schwierigkeit Ausdrücke angeben karın, die den für den Energie-Impulstensor charakteristischen Divergenzrelationen genügen. Die Ausdrücke sind aber nicht eindeutig bestimmt, außerdem führt ihre Berechnung unter Umständen zu komplizierten Grenzübergängen. Überträgt man die Überlegungen, die seinerzeit für die skalare und die vektorielle Theorie von B o p ${ }^{5}$ und dem Verf. ${ }^{9}$ durchgeführt wurden, auf den Spinorfall, so bietet sich als Ausdruck für den kanonischen Tensor $\Theta_{\mu l}$, bzw. den Energie-Impuls-Tensor $T_{\mu v}$ etwa an:

$$
\begin{gathered}
\Theta_{\mu v}=\int d x^{\prime} \int d x^{\prime \prime} \psi\left(x^{\prime}\right) \gamma_{\mu} \psi\left(x^{\prime \prime}\right) \frac{1}{2}\left(\frac{\partial}{\partial x_{v}{ }^{\prime \prime}}-\frac{\partial}{\partial x_{v}^{\prime}}\right) \\
\cdot Z\left(x-x^{\prime}, x-x^{\prime \prime}\right),
\end{gathered}
$$


wobei

$Z\left(x-x^{\prime}, x-x^{\prime \prime}\right)$

$=(2 \pi)^{-8} \int d k^{\prime} \int d k^{\prime \prime} \frac{\zeta\left(k^{\prime}\right)-\zeta\left(k^{\prime \prime}\right)}{-i \gamma_{\tau}\left(k_{\tau}^{\prime}-k_{\tau}^{\prime \prime}\right)} e^{i k^{\prime}\left(x-x^{\prime}\right)-i k^{\prime \prime}\left(x-x^{\prime \prime}\right)}$

$$
T_{\mu v}=\frac{1}{2}\left(\Theta_{\mu v}+\Theta_{v \mu}\right) ; \frac{\partial \Theta_{v \mu}}{\partial x_{v}}=\frac{\partial T_{\mu v}}{\partial x_{v}}=0
$$

Für den Vierervektor von Impuls und Energie folgt aus (11):

$$
\begin{array}{r}
J_{\mu}=\int d \sigma_{\lambda} \int d x^{\prime} \int d x^{\prime \prime} \bar{\psi}\left(x^{\prime}\right) \gamma_{\mu} \psi\left(x^{\prime \prime}\right) \frac{1}{2}\left(\frac{\partial}{\partial x_{j}^{\prime \prime}}-\frac{\partial}{\partial x_{\lambda^{\prime}}}\right) \\
\cdot Z\left(x-x^{\prime}, x-x^{\prime \prime}\right) .
\end{array}
$$

Dabei ist in $x$ über eine raumartige Fläche $\sigma$ zu integrieren.

Das durch Gl. (1) definierte System von Beziehungen entspricht insofern dem alten Hamilton-Formalismus, als es in ihm einen Operator für Gesamtenergie und Gesamtimpuls gibt. Der Begriff Energie- und Impulsdichte verliert jedoch seine einfache frühere Bedeutung, da diese Dichte nicht nur vom Wert der Wellenfunktion an dem betrachteten Ort, sondern auch von ihrem Wert in einer raumzeitlichen Umgebung des Ortes von der Größenordnung $l$ abhängt. In Gebieten, die kleiner sind als die Konstante $l$, kann die Theorie daher im allgemeinen nicht mehr in dem früher üblichen Sinne als eine Nahewirkungstheorie aufgefaßt werden.

Im Spezialfall $\zeta(k)=-\left(i k_{\tau} \gamma_{\tau}+\varkappa\right)$ gehen die Ausdrücke (11)-(13) in die üblichen Ausdrücke für den kanonischen Tensor in der Diracschen Theorie des Elektrons über.

b) Wechselwirkung und Selbstenergie

Wenn eine Wechselwirkung $H(x)$, z. B. von der Form (8), angenommen wird, so kann man ihre Folgen nach den von Tomonaga, Schwinger, Feynman und Dyson angegebenen Methoden berechnen. Man teilt etwa nach dem Vorbild von Schwinger (in der zweiten der angeführten Arbeiten S.652, Gl. 1, 1 a u. b) die Wellenfunktion $\psi_{u}(x)$ in die beiden Anteile

$$
\begin{aligned}
& \psi_{\alpha}(x)=\frac{1}{2}\left(\psi_{\alpha}+(x)+\psi_{\alpha}-(x)\right) \text { ein, } \\
& \psi_{\alpha}^{+}(x)=\frac{1}{2 . \tau i} \int_{c_{+}} \psi_{\alpha}(x-\varepsilon \tau) \frac{d \tau}{\tau}, \\
& \psi_{\alpha}-(x)=\frac{1}{2 . \tau i} \int_{c_{+}} \psi_{\alpha}(x+\varepsilon \tau) \frac{d \tau}{\tau},
\end{aligned}
$$

wobei dann $\psi^{-}(x)$ ein Operator ist, der ein Teilchen (unbekannter Masse!) am Ort $x$ erzeugt, während $\psi^{+}(x)$ ein Teilchen am Ort $x$ beseitigt. Bezeichnet man den Vektor im Hilbert-Raum, der den Zustand Vakuum definiert, mit $\Psi_{0}$, so gilt also

$$
y+(x) \Psi_{0}=0
$$

die konjugierte Gleichung dazu kann man in der Form schreiben

$$
\stackrel{*}{\Psi}_{0} \psi^{-}(x)=0 \text {. }
$$

Allerdings wird der Zustand Vakuum nun modifiziert durch die Wechselwirkung. Für die Behandlung aller Wechselwirkungsfragen ist es am bequemsten, die S-Matrix (9) nach dem Verfahren von Dyson zu berechnen; dabei wollen wir annehmen, daß eine Entwicklung nach Potenzen von $A$ sinnvoll möglich ist.

Der Erwartungswert von $H(x)$ nach Gl. (8) im Zustande $\Psi_{0}$ verschwindet wegen (15) und (16). Also verschwindet die Wechselwirkungsenergie in erster Näherung. Berechnet man aber die zweite Näherung der S-Matrix, so erhält man aus

$$
\int_{-\infty}^{+\infty} d x \int_{-\infty}^{+\infty} d x^{\prime} P\left(H(x), H\left(x^{\prime}\right)\right)
$$

von Null verschiedene Glieder von der allgemeinen Form

$$
A^{2} \int_{-\infty}^{+\infty} d x \int_{-\infty}^{+\infty} d x^{\prime}[\psi+(x)]^{4}\left[\psi^{-}\left(x^{\prime}\right)\right]^{4}
$$

(statt der vierten Potenz steht in Wirklichkeit ein Produkt von vier $\psi(x)$ - oder $\bar{\psi}(x)$-Funktionen und verschiedenen $\gamma$-Operatoren).

Der Erwartungswert von (17) im Zustande $\Psi_{0}$ läßt sich dann nach Dyson zurückführen auf ein Doppelintegral vom Typus

$$
A^{2} \int_{-\infty}^{+\infty} d x \int_{-\infty}^{+\infty} d x^{\prime}\left[S_{F^{\prime}}^{R}\left(x-x^{\prime}\right)\right]^{4},
$$

wobei $S_{F}^{R}\left(x-x^{\prime}\right)$ eine reguläre Vertauschungsfunktion bedeutet, die aus $S_{\alpha \beta}^{R}$ in Gl. (1) in derselben Weise hervorgeht, wie die Feynman-Dysonsche Funktion $S_{F}$ aus der Schwingerschen Funktion $S_{\alpha \beta}$. Die $S^{R}$-Funktionen erlauben alle die gleiche Fourierdarstellung

$$
S^{R}\left(x-x^{\prime}\right)=(2 . \pi)^{-4} \int d k \frac{e^{i k_{\sigma}\left(x-x^{\prime}\right)_{\sigma}}}{\zeta(k)},
$$

wobei zuerst über $k_{4}$ in der komplexen $k_{4}$-Ebene zu integrieren ist, sie unterscheiden sich aber durch den 
Integrationsweg in dieser Ebene. Auf die Einzelheiten der Rechnung, die wir hier nur grob skizziert haben, gehen wir nicht weiter ein, da es sich um eine reine Wiederholung der Rechnungen bei Dyson und Schwinger handeln würde.

Die Gl. (18) berechtigt uns dann zu der Feststellung, daß die Wechselwirkung eine zusätzliche Energiedichte im Vakuum vom Typus

$$
A^{2} \int_{-\infty}^{+\infty} d x^{\prime}\left[S_{F}^{R}\left(x-x^{\prime}\right)\right]^{+}
$$

hervorgebracht hat, die wegen der Regularität von $\mathrm{S}_{F}^{R}$ endlich ist und experimentell nicht weiter in Erscheinung tritt.

Nach dem gleichen Verfahren kann man die Selbstenergie der einzelnen Teilchen ausrechnen. Für den Hilbert-Vektor $\Psi_{1}$ eines Zustandes, in dem nur ein Teilchen vorhanden ist, muß nämlich dann in nullter Näherung gelten:

$$
\psi^{+}(x) \psi^{+}\left(x^{\prime}\right) \Psi_{1}=0 .
$$

Durch ein ähnliches Schlußverfahren, wie oben, erhält man, daß die Selbstenergie erster Ordnung wieder verschwindet und daß in zweiter Näherung aus (17) wieder von Null verschiedene Terme übrig bleiben von der allgemeinen Form

$$
A^{2} \int_{-\infty}^{+\infty} d x \int_{-\infty}^{+\infty} d x^{\prime} \psi-(x)[\psi+(x)]^{3}\left[\psi-\left(x^{\prime}\right)\right]^{3} \psi^{+}\left(x^{\prime}\right) .
$$

Ihre Erwartungswerte geben Ausdrücke vom Typus

$$
A^{2} \int_{-\infty}^{+\infty} d x \int_{-\infty}^{+\infty} d x^{\prime} \psi^{-}(x) \psi^{+}\left(x^{\prime}\right)\left[S_{F^{\prime}}^{R}\left(x-x^{\prime}\right)\right]^{3} .
$$

Der Vergleich mit (11) und (13) lehrt, daß die eigentliche Selbstenergie 2. Ordnung dann gegeben ist durch das Integral über eine raumartige Fläche $\sigma$ :

$$
A^{2} \int_{\sigma} d \sigma \int d x^{\prime} \psi-(x) \psi+\left(x^{\prime}\right)\left[S_{F}^{R}\left(x-x^{\prime}\right)\right]^{3} .
$$

Wegen der Regularität der Funktion $S_{F}^{R}$ ist diese Selbstenergie endlich, und zwar im allgemeinen für jede Teilchensorte verschieden. Die Selbstenergie ist auch nicht etwa einfach der ursprünglichen Masse der Teilchen proportional; denn die Funktion $\left[S_{F}^{R}\left(x-x^{\prime}\right)\right]^{3}$ hängt in einer anderen Weise von $(x-x)$ ab als die Funktion

$$
\int d k \frac{\partial \Xi}{\partial\left(k_{\tau}^{2}\right)} e^{i k_{\sigma}\left(x-x^{\prime}\right)_{\sigma}} .
$$

Schließlich kann nach den Kräften gefragt werden, die zwischen je zwei Teilchen infolge von $H(x)$ wir- ken. Man erhält hier nach dem gleichen Schlußverfahren wie oben in der S-Matrix Ausdrücke 2. Ordnung von der Form:

$A^{2} \int d x \int d x^{\prime} \psi^{-}(x) \psi^{--}\left(x^{\prime}\right)\left[S_{F}^{R}\left(x-x^{\prime}\right)\right]^{2} \psi^{+}(x) \psi^{+}\left(x^{\prime}\right)$,

die uns berechtigen, das Zeitintegral

$$
\int_{-\infty}^{+\infty} d x_{+}^{\prime}\left[S_{F}^{R}\left(x-x^{\prime}\right)\right]^{2}
$$

als das (retardiert zu nehmende) Potential zwischen den beiden Teilchen anzusehen. Dieses Potential verhält sich bei kleinen Abständen regulär und nimmt bei großen Abständen mit der fünften Potenz der Entfernung $a b$, wie man durch eine Untersuchung der betr. Zylinderfunktionen ableitet; es handelt sich also um eine Kraft kurzer Reichweite.

Die Ausdrücke 2. Ordnung, die hier abgeleitet wurden, können natürlich durch Beiträge von höheren Näherungen erheblich modifiziert werden; das Verhältnis der verschiedenen Beiträge hängt vom Absolutwert der Konstante $A$ ab. Bei großen Werten von $A$ wird die Potenzreihenentwicklung nach $A$ überhaupt nicht mehr möglich sein. Man wird aber nach den vorhergehenden Überlegungen erwarten dürfen, daß das ganze System der Gl. (1) und (8) auch dann noch konvergente Resultate liefert.

\section{c) Zusammengesetzte Teilchen}

Die Kraft (24), bzw. ähnliche Ausdrücke höherer Näherung, können dazu führen, daß zwei Spinorteilchen sich zu einem gebundenen Zustand vereinigen, der dann ein Teilchen etwa vom Spin 0 oder 1 repräsentiert. Wenn $A$ klein ist, so wird sich der Hilbert-Vektor $\Psi_{2}$ eines solchen zusammengesetzten Teilchens vom Energie-Impulsvektor $K$ etwa in der Form

$\Psi_{2}=$

$\int d \sigma_{\lambda} K_{i} e^{i\left(K-k-k^{\prime}\right) x_{\tau^{\prime}}}{ }_{\tau}-(k) \psi_{0}-\left(k^{\prime}\right) X_{\tau, 0}\left(k, k^{\prime}\right) d k d k^{\prime} \Psi_{0}$

$[\psi(k)$ ist die Fouriertransformierte von $\psi(x)]$

schreiben lassen, wobei $X_{\tau, g}\left(k, k^{\prime}\right)$ (sozusagen die Eigenfunktion des gebundenen Zustandes im Impulsraum) aus der Bedingung bestimmt werden muß, daß $\Psi_{2}$ einen Eigenzustand der Gesamtenergie bedeutet. $\mathrm{Ob}$ es derartige Lösungen gibt, hängt vom Absolutwert von $A$ und von der speziellen Form von $S_{\alpha \beta}^{R}$ und $H(x)$ ab. Solange $A$ klein ist, bleibt auch die Bindungsenergie des gebundenen Zustandes klein, das zusammengesetzte Teilchen wird dann auch einen verhältnismäßig großen Radius haben. Nur in diesem 
Fall kann man reinlich zwischen einfachen und zusammengesetzten Teilchen scheiden. Der Hilbert-Vektor des einfachen Teilchens hat ja dann die von (26) leicht unterscheidbare Form ${ }^{14}$

$$
\Psi_{1}=\psi^{-}(k) \Psi_{0} .
$$

Wenn jedoch $A$ groß ist, wird die Unterscheidung sinnlos. Denn schon $\Psi_{1}$ enthält dann neben $\psi^{-}(k) \Psi_{0}$ auch Ausdrücke dritten, fünften usw. Grades in $\psi^{-} ; \Psi_{2}$ enthält Ausdrücke vierten, sechsten usw. Grades. Man kann dann zwar noch Teilchen mit ganzzahligem oder halbzahligem Spin unterscheiden, nicht aber mehr zusammengesetzte oder einfache Teilchen. Schon das kräftefreie Elektron der Quantenelektrodynamik kann übrigens in diesem Sinne als ein System aus einem „eigentlichen“ Elektron und einigen Paaren betrachtet werden, die im Abstand $\sim 10^{-11} \mathrm{~cm}$ um das Elektron „umlaufen“, wobei die Bindungsenergie von der Größenordnung $m c^{2}$ ist.

Bei großen Werten von $A$ dürfte es auch vorkommen, daß sich für bestimmte Zustände die Ruhmasse der Spinorteilchen und die von $H(x)$ herrührende Bindungsenergie zu Null kompensieren, so daß ein stationärer Zustand „Lichtquant“ entsteht. Wir glauben, daß die Lichtquanten in dieser Weise aus den Feldgleichungen (1) und (8) folgen müssen, daß man sie also nicht als ein Sonderfeld neben dem Materiefeld einführen sollte. Auch der Wert von $e^{2} / \hbar c$ wäre dann eine unmittelbare Folge der Grundgleichungen vom Typus (1) und (8); in der Tat ist bei dieser Auffassung der relativ kleine Wert von $e / / \hbar c$ durchaus verständlich. Für die Behandlung der elektrodynamischen Vorgänge müßte man ja aus den Matrixelementen der Wechselwirkung (8) nur die spezielle Gruppe von Übergängen herausschneiden, die zur Entstehung (bzw. Vernichtung) eines solchen Zustandes „Lichtquant" führen; daß der Anteil dieser Übergänge nur in der Größenordnung von $1 / 10$ aller Übergänge liegt, erscheint durchaus natürlich.

Der Gedanke, das Lichtquant aus Spinorteilchen zusammenzusetzen, ist übrigens keineswegs neu ${ }^{16}$. Unsere Auffassung unterscheidet sich von den früheren Auffassungen anderer Forscher nur dadurch, daß wir den Zustand Lichtquant nicht durch ein Paar bestimmter Spinorteilchen, sondern durch eine beliebige Anzahl von Paaren aller möglicher Spinorteilchen bilden wollen. Dabei mag ein bestimmtes Paar von

14 Vgl. z. B. R. B e cker u. G. Leibfried, Physic. Rev. 69, 34 [1946].

[1949].
Spinorteilchen in der Schrödinger-Funktion sogar bevorzugt auftreten, aber das ist nicht wesentlich.

In dem gleichen Sinne wäre auch der kürzlich von F e r m i ${ }^{15}$ ausgesprochene Gedanke richtig, daß etwa das $\pi$-Meson als aus Proton und Antiproton zusammengesetzt betrachtet werden sollte. Nur scheint mir der Begriff „zusammengesetzt“" hier zu weit gefaßt, weil dann alle Elementarteilchen ,zusammengesetzt“ wären. Richtig bleibt an ihm nur, daß das $\pi$-Meson ein diskreter Eigenwert desselben Feldgleichungssystems sein muß, das auch die Protonen und alle anderen Elementarteilchen als Eigenwerte liefert.

d) Realitätseigenschaften der Wechselwirkungsfunktion $H(x)$

Die bisherigen Rechnungen wurden so durchgeführt, als ob der Ausdruck für $H(x)$ hermitisch sei. Das ist jedoch im allgemeinen [und insbesondere bei dem als Beispiel gewählten Ausdruck (8)] nicht der Fall. Es ist dem Verf. auch bisher nicht gelungen, einen hermitischen Ausdruck für $H(x)$ zu finden, der aus $\psi$ und $\bar{\psi}$ zusammengesetzt wäre und keine neuen Quellen für Singularitäten enthielte. Es mag sein, daß solche Ausdrücke doch gefunden werden können; dann kann man den Formalismus so eng, wie es oben geschildert wurde, an die traditionellen Methoden der bisherigen Theorie anschließen.

Aber auch wenn man einen nicht-hermitischen Ausdruck wie (8) zugrundelegt, kann man die Theorie zu Ende führen, indem man mit einer naheliegenden Verallgemeinerung der bisherigen Methoden die unitäre S-Matrix bestimmt. Man definiert dann mit Hilfe dieser nicht-hermitischen Funktion $H(x)$ in Analogie zu (9) zunächst eine Matrix $T$ durch die Gleichung .

$$
\begin{aligned}
& T=\sum_{0}^{\infty} \frac{(-i)^{n}}{n !} \int_{-\infty}^{+\infty} d x_{1} \ldots \\
& \int_{-\infty}^{+\infty} d x_{n} P\left(H\left(x_{1}\right), H\left(x_{2}\right) \ldots H\left(x_{n}\right)\right)
\end{aligned}
$$

und setzt

$$
S=T\left(T^{*} T\right)^{-1 / 2}
$$

( $T^{*}$ ist die zu $T$ hermitisch konjugierte Matrix). Die Matrix $S$ ist nun offenbar wieder unitär, wie es sein muß, und kann als die richtige $S$-Matrix des Systems

16 P. J orda n, Z. Physik 102, 243 [1936] u. 105, 114 [1937]; L. de B r o g li e, C. R. hebd. Séances Acad. Sci. 203, 33 [1936] u. 229, 157, 269, 401 [1949]. 
definiert werden. Die Potenzreihenentwicklung von $S$ wird jetzt etwas komplizierter als in Gl. (9). Setzt man

$$
S=\sum_{0}^{\infty} \frac{(-i)^{n}}{n !} S_{n},
$$

so ergibt die Rechnung

$$
\begin{aligned}
S_{1} & =\frac{1}{2} \int_{-\infty}^{+\infty} d x\left(H(x)+H^{*}(x)\right), \\
S_{2} & =\frac{1}{2} \int_{-\infty}^{+\infty} d x_{1} \\
& \int_{-\infty}^{+\infty} d x_{2}\left[P\left(H\left(x_{1}\right), H\left(x_{2}\right)\right)+P\left(H^{*}\left(x_{1}\right), H^{*}\left(x_{2}\right)\right)\right] \\
& -\frac{1}{4}\left\{\int_{-\infty}^{+\infty} d x\left(H(x)-H^{*}(x)\right)\right\}^{2}, \text { u. s. w. }
\end{aligned}
$$

Für eine hermitische Funktion $H(x)$ gehen diese Formeln natürlich wieder in die der gewöhnlichen Theorie über. Dagegen weicht die durch (29) definierte Theorie hinsichtlich der nicht-hermitischen Teile von $H(x)$ erheblich vom kausalen Schema der üblichen Hamilton-Theorie ab. Es handelt sich hier um eine Art von Fernwirkung über raum-zeitliche Bereiche von der Größenordnung der kleinsten Länge, die im normalen Hamilton-Schema nicht beschrieben werden könnte.

In den einzelnen Elementen der S-Matrix kommen neben den Gliedern der Gl. (9), bei deren Berechnung keine Singularitäten auftreten, noch Produkte von derartigen Gliedern vor, von denen man zunächst fürchten könnte, daß sie, da hier über unendlich viele Zwischenzustände summiert wird, zu Divergenzen Anlaß geben. Man muß aber bedenken, daß die Zwischenzustände bei diesen Produkien Zustände von freien Teilchen sind, deren Energiesumme genau gleich der Energie des Anfangs- oder Endzustandes ist. Hierbei treten, ähnlich wie im Formalismus (10), keine Divergenzen auf. Der durch die Gl. (1) bis (8) beschriebene Formalismus läßt sich also trotz des nicht-hermitischen Charakters des Ausdruckes (8) vollständig durchführen.

e) Die Vielfachprozesse und die „Universalmischung " der Elementarteilchen

Das empirische Spektrum der Elementarteilchen bildet ein sehr starkes Argument für die An- nahme, daß in dem richtigen System der Feldgleichungen die der Größe $A$ entsprechende Konstante einen ziemlich großen Wert hat $\left(A \gtrsim l^{2}\right)$. Diese Voraussetzung hat, wie schon früher ausgeführt ${ }^{17}$, folgende wichtige Konsequenz: Lokalisiert man einen sehr großen Energiebetrag $(\gg \hbar c / l)$ in einem sehr kleinen Raumgebiet (von der Größenordnung der „,kleinsten Länge“), so stellt sich nach kürzester Zeit infolge der turbulenten Durchmischung durch die große nichtlineare Wechselwirkung eine „Universalmischung" von Materie ein, die im wesentlichen durch die gesamte zur Verfügung stehende Energie bestimmt ist und nur wenig von den speziellen Anfangsbedingungen abhängen dürfte. Die Häufigkeit der dabei entstehenden verschiedenen Sorten von Elementarteilchen, ihre Anzahl und Richtungsverteilung, wird also praktisch nur von der Gesamtenergie abhängen, und es wird gleichgültig sein, welche Elementarteilchen zusammengestoßen sind, um diese Energie an einem Punkt zu konzentrieren. Daß diese Unabhängigkeit vom Primärprozeß tatsächlich aus einem Formalismus mit einem hohen A-Wert folgt, kann auch leicht an einem Beispiel genauer nachgerechnet werden, das im Zusammenhang mit der $S$-Matrixtheorie früher untersucht worden ist (in der zweiten unter ${ }^{9}$ erwähnten Arbeit S. 691 bis 698); die Einzelheiten sollen aber hier nicht ausgeführt werden.

Auch die empirischen Tatsachen sprechen für die Existenz der Vielfachprozesse und damit der „Universalmischung", wie früher in anderen Arbeiten erörtert worden ist ${ }^{17}$.

Der Ansatz (8) für die Wechselwirkung ist natürlich nur ein sehr spezielles Beispiel aus einer viel größeren Gruppe von möglichen Ausdrücken für $H(x)$. Selbst wenn man sich auf eine Wechselwirkung beschränkt, die in der Wellenfunktion vom vierten Grade ist, könnte man z. B. einen Ausdruck vom Typus

$$
\begin{gathered}
A \int d x \int d x^{\prime} \int d x^{\prime \prime} \int d x^{\prime \prime \prime} f\left(x-x^{\prime}, x-x^{\prime \prime}, x-x^{\prime \prime \prime}\right) \\
\psi(x) \psi\left(x^{\prime}\right) \bar{\psi}\left(x^{\prime \prime}\right) \psi\left(x^{\prime \prime \prime}\right)
\end{gathered}
$$

wählen, in den eine zunächst beliebige Funktion $f$ von drei Variabeln eingeht. An den vorher skizzierten Rechnungen würde sich dabei nichts Wesentliches ändern.

Die Geschlossenheit und prinzipielle Einfachheit eines durch die Ausdrücke $S_{\alpha, \beta}^{R}\left(x-x^{\prime}\right)$ und $H(x)$

17 W. He is enberg, Z. Physik 113, 61 [1939]; G. W a t a g h in, Physic. Rev. 74, 975 [1948]; W. H e i s e n berg, Z. Physik 126, 569 [1949]. 
definierten Gleichungssystems scheinen dem Verf. ein starkes Argument dafür, daß die wirkliche Theorie der Elementarteilchen durch einen solchen Formalismus dargestellt wird. Die große Mannigfaltigkeit der Elementarteilchen würde damit auf ein einfaches Gleichungssystem zurückgeführt, in dem als Dimensionskonstante nur noch die „kleinste Länge“ $l$ vorkommt. Alle Elementarteilchen wären nur stationäre Zustände ein und derselben „Materie“.

Unbefriedigend bleibt einstweilen noch die große Willkür, die bei der Wahl der Funktionen $S_{\alpha \beta}^{R}$ und $H(x)$ zu bestehen scheint; aber man kann hoffen, daß die richtigen Funktionen durch besondere Eigenschaften, etwa durch Einfachheit oder durch Invarianz bei bestimmten Transformationen, ausgezeichnet sind. Die Aufgabe der nächsten Zeit wird eben darin bestehen, aus den empirischen Eigenschaften der Elementarteilchen Schlüsse auf die Strukturen der Funktionen $S_{\alpha \beta}^{R}$ und $H$ zu ziehen. Zum Verständnis dieser Strukturen gehört z. B. auch das Verständnis der Eichtransformation und des mit ihr offenbar verbundenen Wertes von $e^{2} / \hbar c$; denn die ganze Quantenelektrodynamik sollte ja nur ein Ausschnitt aus dem Gleichungssystem (1) und (8) sein.

\title{
Über die Konzentrationsabhängigkeit von Volumeneffekt und Dipolmoment binärer und ternärer Gemische einiger organischer Flüssigkeiten
}

\author{
Von Maria Joerges, geb. Heyden und Alexander Nikuradse \\ Aus dem Institut für Elektronen- und Ionenforschung an der Technischen Hochschule München \\ (Z. Naturforschg. 5 a, 259-269 [1950]; eingegangen am 9. Februar 1950)
}

An binären und ternären Gemischen von Hexan, Methylalkohol und Chlorbenzol sowie von Schwefelkohlenstoff, Methylalkohol und Aceton werden Volumeneffekt und Dielektrizitätskonstante bei $20^{\circ} \mathrm{C}$ bestimmt. Aus den DK-Messungen werden nach der Debye-ClausiusMosottischen und nach der Onsagerschen Formel die Dipolmomente berechnet. Aus ihrer Konzentrationsabhängigkeit und dem Verlauf des Volumeneffektes wird auf den Assoziationszustand geschlossen.

Speziell läßt sich für Aceton-Methylalkoholgemische aus dem nach Onsager berechneten Dipolmoment eine $\mathrm{OH} \ldots \mathrm{O}=\mathrm{C}$-Brückenbildung ablesen.

$\mathrm{D}$ er Volumeneffekt besteht in einer VolumenKontraktion oder -Dilatation, die meist beim Mischen von Flüssigkeiten eintritt. Die Dielektrizitätskonstante einer Substanz ist eine Funktion der Polarisierbarkeit, deren Größe und Verhalten vor allem dann von Interesse ist, wenn es sich um Moleküle handelt, die ein permanentes elektrisches Moment tragen. Untersucht man nun an den gleichen Gemischen organischer Flüssigkeiten Dielektrizitätskonstante und Volumeneffekt, so steht zur Diskussion, ob und in welcher Weise diese beiden Funktionen molekularer Eigenschaften miteinander verknüpft sind. Schon früher sind darüber von K. L. Wolf und Mitarbb. Úberlegungen angestellt worden. Sie stützten sich aber hinsichtlich des Verhaltens der polaren Substanzen lediglich auf die Debyesche Theorie und die Debye-Clausius-Mosottische Formel zur Berechnung von Dipolmomenten gasförmiger Substanzen. Es konnte sich daher keine allgemein befriedigende Deutung ergeben. Die von Onsager und später von $\mathrm{K}$ irkw o od speziell für das flüssige Dielektrikum entwickelte Theorie, die früher wenig beachtet und erst in jüngster Zeit durch die Arbeiten von M e cke und seinen Schülern aufgegriffen und eingehender Kritik unterworfen wurde, hat ergeben, daß sie gerade dann von besonderer Bedeutung ist, wenn es sich nicht um die immer zwischen Dipolen auftretenden Attraktionskräfte handelt, sondern um eine spezifische, also strukturabhängige zwischenmolekulare Wechselwirkung gleicher oder ungleicher Moleküle untereinander.

$\mathrm{Zu}$ den im folgenden berichteten Untersuchunge: wurden binäre und ternäre Gemische aus Schwefelkohlenstoff, Aceton und Methylalkohol und außerdem Gemische aus Hexan, Chlorbenzol und Methylalkohol herangezogen. Von ihnen sind bekanntlich Aceton, Chlorbenzol und Methylalkohol Dipolsubstanzen, während Schwefelkohlenstoff und Hexan unpolare Flüssigkeiten sind. 